\title{
Flow cytometric analyses of the viability, surface marker expression and function of lymphocytes from children following cryopreservation
}

\author{
XI CHEN $^{1,2^{*}}$, HUI ZHANG ${ }^{1,2^{*}}$, WENJUN MOU ${ }^{1,2}$, ZHAN QI $^{1,3}$, XIAOYA REN $^{1,4}$, GUOLIANG WANG $^{1,2}$, \\ HONG JIAO $^{1,2}$, XIAOHUI KONG ${ }^{1,2}$ and JINGANG GUI ${ }^{1,2}$ \\ ${ }^{1}$ Key Laboratory of Major Diseases in Children by Ministry of Education, ${ }^{2}$ Laboratory of Immunology; \\ ${ }^{3}$ Center for Medical Genetics, Beijing Pediatric Research Institute; ${ }^{4}$ Department of Pediatric Endocrinology and Genetic Metabolism, \\ Beijing Children's Hospital affiliated with Capital Medical University, Beijing 100045, P.R. China
}

Received September 7, 2015; Accepted September 7, 2016

DOI: $10.3892 / \mathrm{mmr} .2016 .5780$

\begin{abstract}
Flow cytometric analysis is important for the investigation and clinical preparation of lymphocytes from children. However, the strict requirement of cell freshness and inter-assay variability limits the application of this methodology for pediatric investigations. Therefore, it is necessary to identify a reliable cryopreservative method capable of maintaining high cell viability and proper cell function in lymphocytes from children. In the present study, eight commonly-used cell cyropreservative methods were used, and their effects on cell viability, surface marker expression and cell function were examined. In addition, how these methods affect the distribution of T-cell receptor $\mathrm{V} \beta$ subfamilies were also determined. The results of the present study provided valuable experimental evidence, based on which the optimal method for the cryopreservation of lymphocytes from children in pediatric investigations and clinical applications can be selected.
\end{abstract}

\section{Introduction}

Flow cytometry is a key approach for immunological investigations, providing a convenient method to detect cell differentiation markers, cytokines, transcriptional factors and DNA content, in a number situations, simultaneously (1). It has also been frequently used for the analyses of cell function,

Correspondence to: Professor Jingang Gui, Laboratory of Immunology, Beijing Pediatric Research Institute, Beijing Children's Hospital affiliated with Capital Medical University, 56 Nan-li-shi Road, Beijing 100045, P.R. China

E-mail: guijingang@bch.com.cn

*Contributed equally

Key words: flow cytometry, children, lymphocytes, cryopreservation, cytokines, T-cell receptor repertoire cytokine expression, cell apoptosis and proliferation. However, in the majority of cases, flow cytometric analysis has a strict requirement for fresh samples. Cell death not only alters the expression level/pattern of cell surface markers, which is critical for the accuracy of cell subpopulation determination, but also affects the ex vivo cell function, which is closest to the in vivo status experiments are aiming to recreate. This strict time requirement causes limitations for investigations, particularly clinical investigations, in two respects. Firstly, it requires that samples are processed within a short time period without delay. This problem is worsened if patient surgery or other treatments are arranged later in the day. Secondly, without an optimal protocol to preserve the sample, experiments are performed discretely. As a result, the data collected are of increased variance due to the unavoidable difference generated from independent experiments. The inter-assay variance becomes more marked in certain complex experiments, including cell stimulation in culture followed by intracellular cytokine measurement.

Clinical trials or approved cell therapy, particularly immunotherapy, require a large number of viable functional cells. For example, the availability of large quantities of functionally effective dendritic cells for immunotherapeutic trials against infectious diseases is critical for the effectiveness of cell therapy $(2,3)$. The infusion of genetically engineered T-cells with chimeric antigen receptors for cancer therapy involves concentrated cell preparations (4). Another example is the storage of cord blood, a valuable source of hematopoietic stem cells for the treatment of several serious diseases (5).

Therefore, the cryopreservation of immune cells is indispensable for experimental and clinical use. Different cryoprotectants, cytomedia additives and freezing procedures are continuously being assessed to optimize cell cryopreservation for different purposes (6-9). The primary aim is to protect cells from the adverse effects of the ice crystals formed, which either completely destroys cells or eventually affects cell viability and function.

As an effective cyroprotective formula, $10 \%$ dimethyl sulfoxide (DMSO) in $90 \%$ fetal bovine serum (FBS) has been 
widely used, particularly for experimental purposes due to the xenogenic property of FBS to humans and possible zoonotic contamination. Unlike traditional pooled human serum, which may contain viral or other bioactive contamination, human serum albumin (HSA) combined with DMSO is frequently selected as a standard protocol for clinical use (10).

Lymphocytes in children are different from those of adults in terms of their subset proportions, cell functions and their responsiveness to antigens $(11,12)$. How cryopreservation affects lymphocytes of children remains to be elucidated. In the present study, alterations in cell viability, subset proportion, cytokine production and $\mathrm{T}$ cell receptor (TCR) V $\beta$ subfamily distribution were examined following the thawing of cells from eight cryopreservation methods. The results aimed to provide a valuable reference for the optimal storage of blood cells from children for pediatric investigations and clinical applications.

\section{Materials and methods}

Ethics statement. The present study was performed according to the principles expressed in the Declaration of Helsinki and was approved by the Ethics Committee of the Beijing Children's Hospital, Capital Medical University (Beijing, China). Whole blood samples from 80 children aged 1-6 years old (38 female, 42 male) were collected in Beijing Children's Hospital following the provision of written informed consent for its use for experimental purposes from the children's parents or guardians.

Blood sample collection. Patients at Beijing Children's Hospital were recruited to the present study between June 2014 and June 2015. Patients with immune system-associated diseases or diseases affecting lymphocyte proportion and function were excluded. All blood samples were collected in EDTA blood collection tubes. The experiments were performed at least three times to reduce single operating error and sample variation. Within each cryopreservative method, comparisons were made for the same sample between data collected prior to freezing and that collected post-thawing.

Cell freezing and thawing. In method 1, the whole blood sample was not pre-treated prior to freezing. In method 2, DMSO (1:10 total blood volume) was added to the whole blood prior to freezing. In methods 3 and 4, red blood cells (RBCs) were lysed with RBC lysis buffer (OptiLyse C lysis solution; Beckman Coulter, Miami, FL, USA). In methods 7 and 8 , the RBCs were lysed with ammonium chloride lysing solution (10X stock) containing $1.5 \mathrm{M} \mathrm{NH}_{4} \mathrm{Cl}, 100 \mathrm{mM}$ $\mathrm{NaHCO}_{3}$ and $10 \mathrm{mM}$ EDTA-Na $(\mathrm{pH}$ 7.4). The cells were spun for $5 \mathrm{~min}$ at $500 \mathrm{RCF}$. The cell pellets were then washed once with RPMI medium prior to being resuspended with $10 \%$ DMSO+90\% FBS or with $10 \%$ DMSO+90\% HSA, respectively. In methods 5 and 6 , lymphocytes were purified from the whole blood by density gradient centrifugation (1000 x g, $20 \mathrm{~min}$, room temperature) over lymphocyte separation medium. The cells were then washed and resuspended with $10 \%$ DMSO +90\% FBS or with $10 \%$ DMSO+90\% HSA as cryoprotective additives, respectively. The cells in the cryovials were first stored at $-80^{\circ} \mathrm{C}$ for 3 days in a Nalgene cell freezing container (Thermo Fisher Scientific, Inc., Watlham, MA, USA) filled with isopropanol, and then moved to a $-196^{\circ} \mathrm{C}$ liquid nitrogen tank for long-term storage. Cell thawing was performed by removing the frozen vial from the liquid nitrogen tank and immediately immersing it into a $37^{\circ} \mathrm{C}$ water bath for $\sim 5 \mathrm{~min}$ with intermittent agitation. The thawed cells were washed once with PBS and resuspended with cell staining buffer or cell culture medium for the respective experiments.

Cell count and viability assessment. The absolute cell count was determined using a Millipore Guava Easycyte 8 flow cytometer (EMD Millipore, Billerica, MA, USA). In brief, with appropriate adjustment of FSC (47.3 V) and SSC (108 V) voltages, granulocytes, monocytes and lymphocytes were well separated. Targeting a total of 5,000 lymphocyte-gated events, the flow cytometry recorded a volume of sample consumed, and the number of lymphocytes (number/ $\mu 1$ of loaded sample) was calculated. The dilution factor (10) was then applied to obtain the total lymphocyte cell count (cells/ml blood). The sample was stained with 7-amino-actinomycin D (7-AAD) to distinguish between the viable (7-AAD $)$ and dead cells $\left(7-\mathrm{AAD}^{+}\right)$.

Flow cytometric analysis. The fluorochrome-conjugated mouse anti-human antibodies: Fluorescein isothiocyanate (FITC)-CD8, FITC-CD16, phycoerythrin (PE)-CD4, PE-interleukin (IL)-2, PEcy5-CD3, PEcy5-CD56, PEcy5-CD19 and antigen presenting cell-interfron $\gamma$ (APC-IFN $\gamma$ ) were purchased from BioLegend, Inc. (San Diego, CA, USA). Incubation was conducted for at $4^{\circ} \mathrm{C}$. For surface staining, cells prior to freezing and after thawing, and those harvested from cell culture were washed once with PBS, and stained with antibodies in cell staining buffer (3\% FBS in PBS) in the dark for $25 \mathrm{~min}$. For intracellular staining, the Cytofix/Cytoperm ${ }^{\mathrm{TM}}$ Fixation/Permeabilization kit (BD Biosciences, San Diego, CA, USA) was used according to the manufacturer's protocol. For determining the TCR V $\beta$ repertoire, the IO Test Beta Mark V $\beta$-TCR repertoire kit (Beckman Coulter) was used. FlowJo software, version 7.6 (Tree Star, Inc., Ashland, OR, USA) was used for flow cytometric data analysis.

Cell culture and lymphocyte activation. A U-bottom 96-well culture plate was coated with $10 \mu \mathrm{g} / \mathrm{ml}$ solution of anti-CD3 in sterile PBS and maintained at $4^{\circ} \mathrm{C}$ overnight. The cells (1x10\% well) were cultured for $20 \mathrm{~h}$ at $37^{\circ} \mathrm{C}$ with $5 \% \mathrm{CO}_{2}$ in complete RPMI 1640 medium (200 $\mu \mathrm{l} /$ well) supplemented with $10 \%$ FBS, 2 mM L-glutamine, $1 \%$ penicillin/streptomycin, $5 \mathrm{ng} / \mathrm{ml} \mathrm{IL-2}$ and anti-CD28 $(2.5 \mu \mathrm{g} / \mathrm{ml})$-free antibodies. Final lymphocyte activation was performed by adding PMA $(50 \mathrm{ng} / \mathrm{ml})$ and ionomycin $(1 \mu \mathrm{g} / \mathrm{ml})$ in the presence of GolgiStop (monensin; $2.5 \mu \mathrm{l} / \mathrm{ml}$; BD Biosciences). The culture was maintained in the incubator for another $5 \mathrm{~h}$. The cells were then washed twice with staining buffer prior to surface and intracellular staining with PE-IL-2 and APC-IFN $\gamma$.

Statistical analysis. A paired t-test was performed to compare the differences between each fresh blood sample and its corresponding thawed frozen sample in terms of surface 
marker and intracellular cytokine expression following activation. GraphPad Prism software, version 5 (GraphPad, Inc., La Jolla, CA, USA) was used for statistical analysis. $\mathrm{P}<0.05$ was considered to indicate a statistically significant difference.

\section{Results}

Viability maintenance of lymphocytes cryopreserved using different methods. In the present study, eight methods for the cryopreservation of blood cells from children were compared. As expected, the whole blood cells frozen directly without any cryoprotective additive exhibited complete loss of the typical cytometric pattern comprising the three major cell populations of granulocytes, monocytes and lymphocytes, based on FSC, vs. SSC values in flow cytometry (Fig. 1). The addition of DMSO only to the whole blood prior to freezing in method 2 resulted in the thawed cells exhibiting a similar pattern to those frozen using method 1 . The three major cell populations were well maintained in the cells cryopreserved with methods 3, 4, 7 and 8, in which the RBCs were lysed first (hemolysin for methods 3 and 4; $\mathrm{NH}_{4} \mathrm{Cl}$ for methods 7 and 8) followed by the addition of standard medium (90\% FBS+10\% DMSO for methods 3 and 7; 90\% HSA $+10 \%$ DMSO for methods 4 and 8) prior to freezing. There was an appreciable decrease in SSC values for the granulocyte population. In methods 5 and 6 , in which the lymphocytes were isolated by lymphocyte separation medium prior to being frozen with $10 \%$ DMSO+90\% FBS or HSA, respectively, no alterations in the FSC or SSC values of the lymphocyte population were observed, compared with the same sample fresh following collection.

Measuring the absolute number of viable lymphocytes in the same sample prior to and following freezing revealed that methods 1 and 2 resulted in a marked cell death (Fig. 2). Following thawing from method 1 , the remaining viable (7-AAD ${ }^{-}$) lymphocytes were $40 \% \pm 13$ (mean \pm standard error of the mean) of the lymphocytes measured in the fresh sample. Method 2 was the least effective among the cryopreservative methods in terms of their ability to maintain lymphocyte viability. The percentage of remaining lymphocytes relative to the fresh sample was only $25 \% \pm 13$. The remaining methods (methods 3-8) maintained a mean viability of lymphocytes between 59 and $77 \%$, relative to their respective cell counts determined prior to freezing. Among these, method 5 provided the optimal lymphocyte protection, with a viability of $77 \% \pm 10$. However, the lymphocyte counts prior to freezing with methods 5 and 6 decreased by $50 \%$, caused by the lymphocytes isolation procedure, compared with the lymphocyte count in the same sample measured without purification. Therefore, a high ratio of thawed viable lymphocytes to their counterparts prior to freezing does not necessarily indicate a high absolute lymphocyte count in the thawed samples.

Changes in the percentage of the lymphocyte subpopulations following cryopreservation. The present study then aimed to determine how the different methods of cryopreservation affected the percentages of lymphocyte subpopulations. As shown in Fig. 3, methods 1 and 2 resulted in a significant decrease in the percentages of $\mathrm{CD}^{+} \mathrm{T}$-cells and $\mathrm{CD} 19^{+} \mathrm{B}$-cells in the thawed cells, in the context of a marked decline in viable lymphocytes. The percentage of $\mathrm{CD} 16^{+} \mathrm{CD} 56^{+} \mathrm{NK}$ cells in method 1 was increased in lymphocyte gates, whereas the average percentage of NK cells in method 2 decreased, but not significantly. Method 3 resulted in a significant decrease in the percentage of $\mathrm{CD} 19^{+} \mathrm{B}$ cells, but not $\mathrm{CD}^{+} \mathrm{T}$ cells, compared with the measurements in the fresh sample. Method 4 exhibited a decrease in the percentages of $\mathrm{B}$ and $\mathrm{T}$ cells, however, this was not significant. By contrast, methods 3 and 4 led to a statistically significant increase in the percentage of NK cells. Method 5 also led to a significant alteration in the percentage of $\mathrm{B}$ and $\mathrm{T}$ cells, however, this was in the opposite direction. The percentage of $\mathrm{B}$ cells decreased, but that of $\mathrm{T}$ cells increased, compared with their respective values measured prior to freezing. By contrast, the percentage of NK cells was not altered by method 5. Methods 6-8 maintained a broad spectrum of lymphocyte subpopulations, as they did not affect the percentage of B cells, NK cells or T cells, with the exception of method 7, which showed a significant increase in $\mathrm{T}$ cells, resembling that observed in method 5 .

The present study then investigated whether CD4 and CD8 T cells are differentially affected by cryopreservation, which may lead to a percentage change in the $\mathrm{CD}^{+} \mathrm{T}$ cell gate. The data indicated that CD4 and CD8, in the majority of the methods, were proportionally affected. As shown in the lower panel of Fig. 3, the relative CD4 or CD8 percentage in the $\mathrm{CD}^{+} \mathrm{T}$ cell population remained unaltered in methods 1 , 2, 3, 4, 7 and 8. Significant alterations existed in methods 5 and 6 , of which the former caused a decrease in CD8 cells and the latter caused a decrease in CD4 T cells.

Functional changes of lymphocytes following cryopreservation. To determine whether there is an optimal freezing method for the maximum maintenance of function of lymphocytes from children, the present study determined the intracellular expression of IFN $\gamma$ and IL-2 in the lymphocytes of children being cryopreserved, the expression of which is the hallmark of activated lymphocyte function. Following stimulation of the thawed cells with anti-CD3/CD28 in the presence of PMA and ionomycin in culture, no intracellular expression of IFN $\gamma$ or IL-2 were detected in the cells cryopreserved using methods 1 and 2 due to extensive cell death. Upon stimulation of the cells thawed from methods 3 and 4, which were confirmed to have considerable viability, did not express intracellular IFN $\gamma$ or IL-2. By contrast, cytokines were successfully detected in the purified lymphocytes thawed from methods 5 and 6 , and those cryopreserved using methods 7 and 8 in which RBCs were lysed with $\mathrm{NH}_{4} \mathrm{Cl}$ (Fig. 4). In methods 5-8, although the percentage of IFN $\gamma$ relative to its respective value in the fresh sample varied between experiments, the mean fluorescence index (MFI) was invariably reduced in all independent experiments. By contrast, the percentage and MFI of IL-2 were consistently decreased following cryopreservation in every independent experiment, with method 8 as an exception. The percentage and MFI of the intracellular staining of IL-2 in method 8 were bidirectionally variable between experiments.

Altered TCR V $\beta$ subfamily distribution following cryopreservation. The measurement of bias-usage or diversity changes of the 


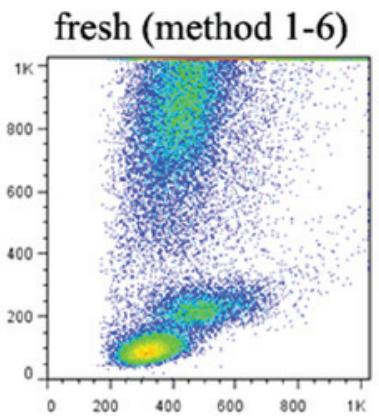

method 3

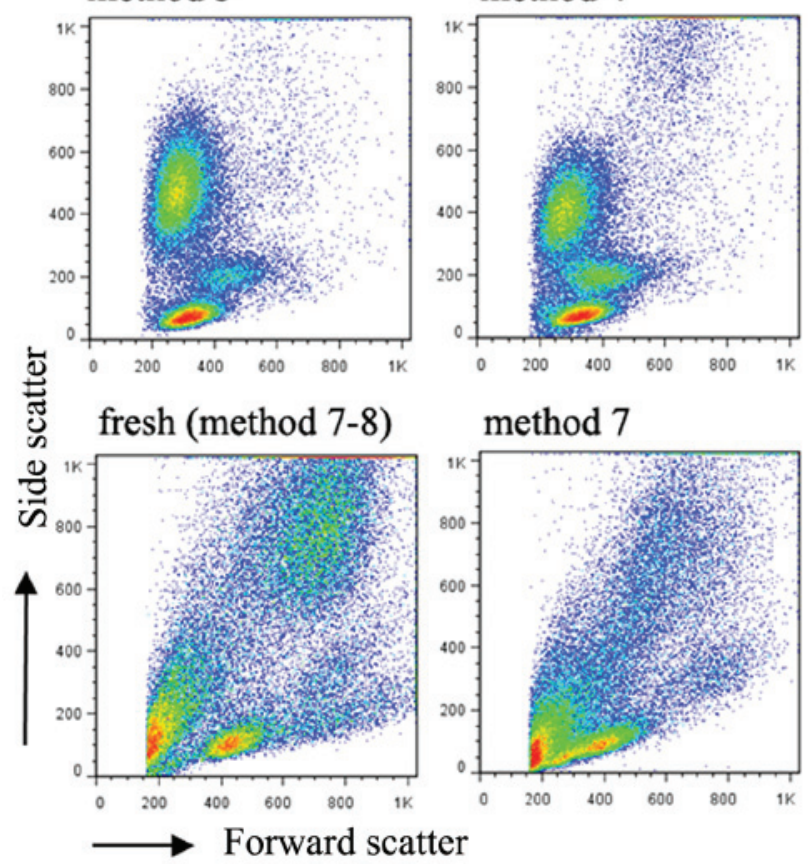

method 1

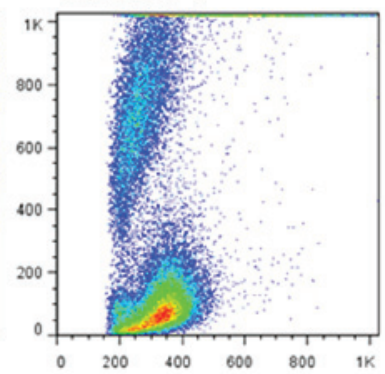

method 4

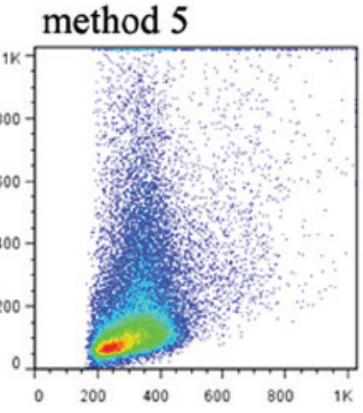

method 6

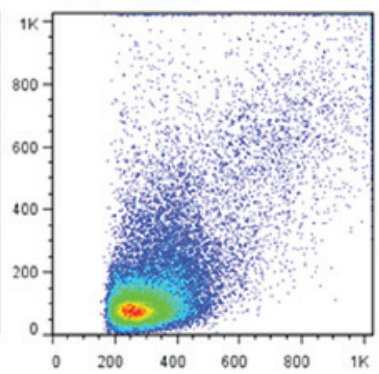

method 8

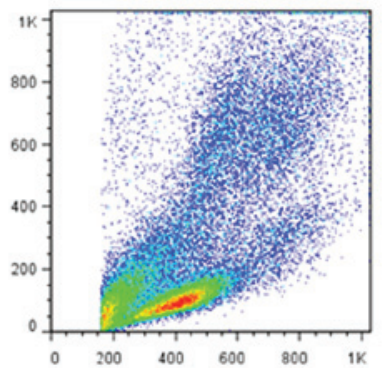

Figure 1. FSC and SSC pattern of blood samples following cryopreservation. Red blood cells in blood sample were lysed in OptiLyse C lysis solution and cells were collected immediately as fresh data using a Beckman Coulter Epics XL flow cytometer. The remaining blood was treated and cryopreserved using methods 1-6. Thawed samples from methods 1-6 were collected using the same flow cytometer and the same FSC and SSC voltage as used to collect the fresh sample. Blood samples used for methods 7 and 8 differed from methods 1-6. The flow data for methods 7 and 8 and their corresponding flow data prior to freezing (fresh) were generated using a BD FACScalibur flow cytometer with the same FSC and SSC settings. FSC, forward scatter; SSC, side scatter.

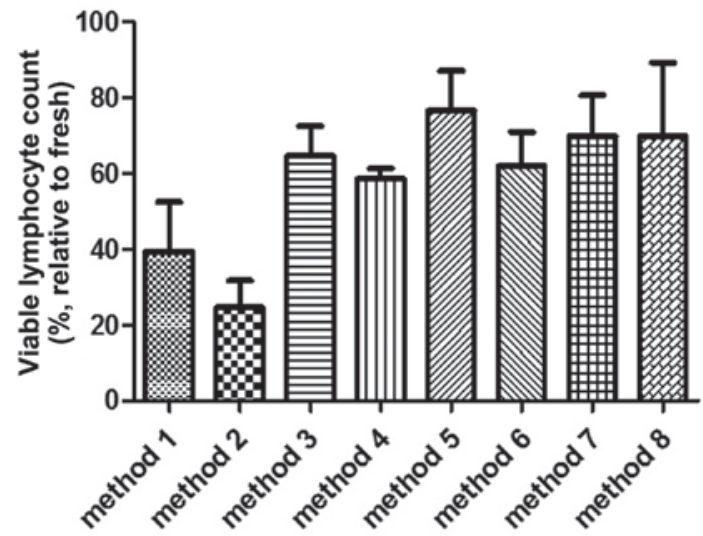

Figure 2. Viability of lymphocytes in blood samples following cryopreservation. Red blood cells in the blood samples were lysed in OptiLyse C lysis solution and the total viable lymphocyte counts were measured using a Guava Easycyte 8 flow cytometer. Viable lymphocyte counts in the thawed samples, cryopreserved using different methods were measured 10 days later and expressed as a percentage relative to the respective lymphocyte count prior to freezing (fresh). Each bar is based on four independent experiments, with at least four blood samples collected for each method in each independent experiment. Data are presented as the mean + standard error of the mean.
TCR V $\beta$ repertoire as an immune characteristic has been documented in several diseases and malignant conditions (13-16). The results of the present study revealed that freezing differentially affected cell death in different subsets, which caused percentage changes in the lymphocyte subpopulations. The present study then investigated whether any of the cryopreservative methods supported the TCR V $\beta$ staining without causing a differential change in the $24 \mathrm{~V} \beta$ subfamilies. The preferential alteration of particular subfamilies of $\mathrm{V} \beta$ by cryopreservation results in a change in $\mathrm{V} \beta$ subfamily distribution, which leads to an error in assessing the TCR repertoire (data not shown). To assess this, the present study compared the percentages of $24 \mathrm{~V} \beta$ subfamilies in the blood samples prior to freezing, and these values were monitored following cryopreserved of the cells using methods $3-8$. The cells directly preserved without cryoprotective additives or with the addition of DMSO only in methods 1 and 2, respectively were found to cause extensive cell death. Therefore, the antibody staining of TCR V $\beta$ stored in cells in these two methods was omitted. The data collected from methods 3-8, as shown in Fig. 5, were classified into three categories based on the change in $V \beta$ subfamilies following cyropreservation. 

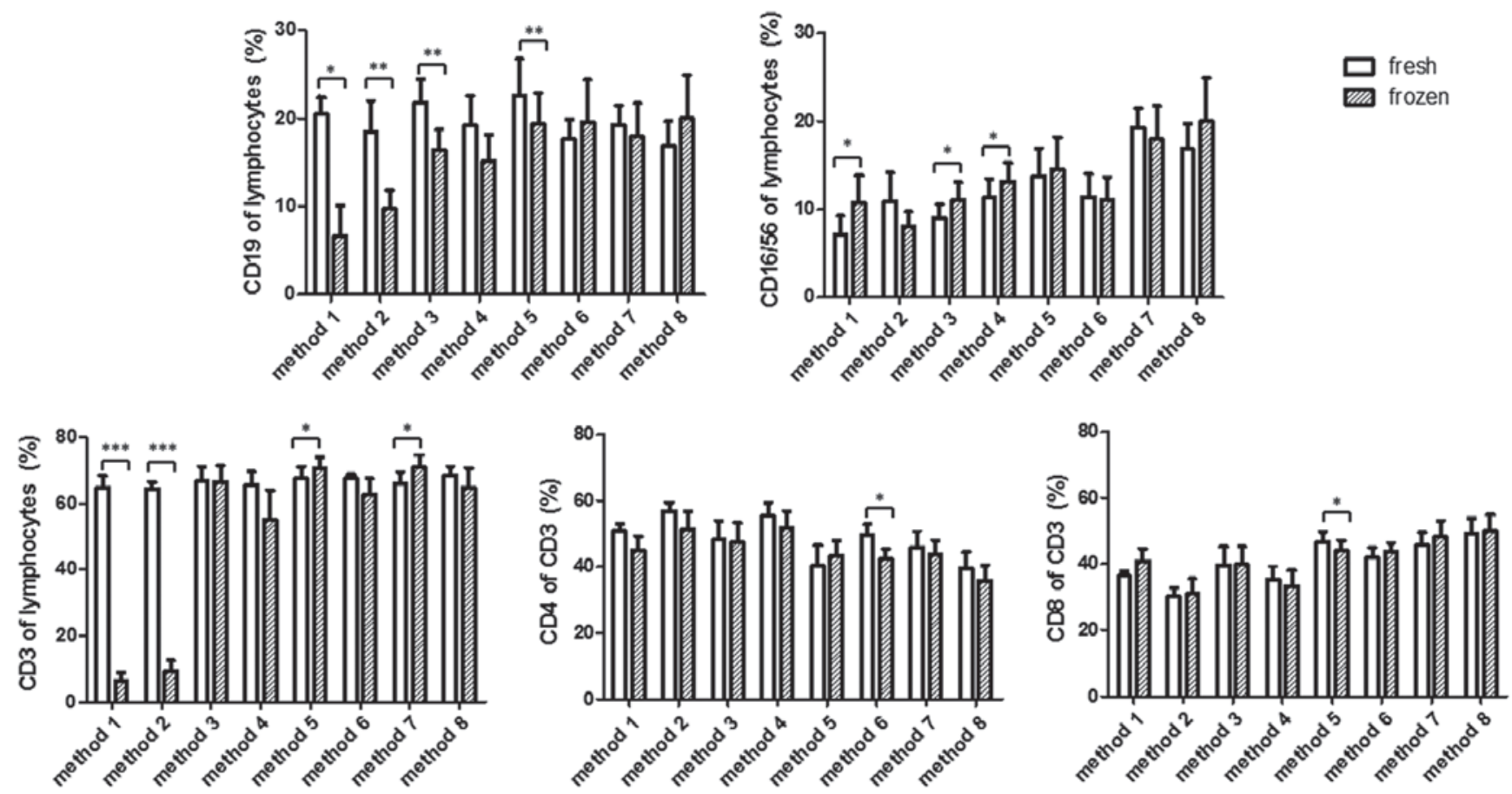

Figure 3. Surface CD marker expression of lymphocytes in blood samples following cryopreservation. Blood samples were cryopreserved using different methods. The percentages of $\mathrm{CD} 19^{+} \mathrm{B}$ cells, $\mathrm{CD}^{+} \mathrm{CD}^{+}$and $\mathrm{CD}^{+} \mathrm{CD} 8^{+} \mathrm{T}$ cells, and $\mathrm{CD} 16^{+} \mathrm{CD} 56^{+}$natural killer cells following thawing (frozen) are shown compares with the percentage prior to freezing (fresh). Raw flow cytometry data were collected using a BD FACScalibur flow cytometer and analyzed using FlowJo software. Paired $t$-tests were performed to compare differences between the fresh and frozen samples. ${ }^{*} \mathrm{P}<0.05 ;{ }^{* *} \mathrm{P}<0.01 ;{ }^{* * *} \mathrm{P}<0.001$. Data are represented as the mean + standard error of the mean. Each bar is based on five independent experiments. At least three blood samples were collected for each method in each independent experiment.

Firstly, the percentage of V $\beta$ subfamilies were decreased by all cryopreservative methods. These $\mathrm{V} \beta$ subfamilies included $\mathrm{V} \beta$ 5.2, V $\beta 9$ and V $\beta 22$ (Fig. 5A). Secondly, changes in the percentages of certain $\mathrm{V} \beta$ subfamilies were variable in a method-dependent manner. These included V $\beta$ 5.1, 13.2, 14, 16, 18, 20 and $\mathrm{V} \beta 23$ (Fig. 5B). Thirdly, the percentages of certain $\mathrm{V} \beta$ subfamilies were not affected by any of the cryopreservative methods (Fig. 5C). These subfamilies included V $\beta$ 1, 2, 3, 4, 5.3, 7.1, 7.2, 8, 11, 12, 13.1, 13.6, 17 and 21.3.

\section{Discussion}

The ability to store lymphocytes, and maintain sufficient viability and function has been an important issue for investigations and clinical applications. The present study compared several commonly used cryopreservation methods for the efficient storage of lymphocytes of children, which differs from that of adults with regard to the cell function and, possibly, susceptibility to freezing. The data based on methods 1 and 2 showed that direct freezing of whole blood samples resulted in extensive cell death. As shown in the FSC, vs. SSC flow plots in Fig. 1, adding DMSO as a cyroprotective additive to the whole blood exacerbated the cell death in cryopreservation. It is likely that salt and/or other unknown small molecules in the plasma of the whole blood contributed to the dominant detrimental effect in the freeze-thaw cycle. The direct addition of $10 \%$ DMSO in the context of insufficient endogenous human serum, in which the endogenous human serum was $<90 \%$ of the total volume as suggested in the frozen medium formula, provided no beneficial effect, and was toxic to cells. When the leukocytes in the whole blood were washed with PBS and resuspended with $10 \%$ DMSO+90\% FBS or HSA in methods 3,4,7 and 8, the thawed cells largely maintained their viability (Fig. 2). Of note, an appreciable decrease in the granulocyte SSC values in methods 3, 4, 7 and 8 (Fig. 1) suggested a change in the internal complexity of granulocytes as a result of freezing.

The changes in the percentage of lymphocyte subpopulations in the present study suggested that, in certain situations (Fig. 3; methods 3 and 5), CD19 ${ }^{+} \mathrm{B}$ cells were more susceptible to freezing damage, compared with $\mathrm{CD}^{+} \mathrm{T}$ cells. The fact that the percentage of NK cells increased in methods 3 and 4 is possibly a reflection of relative decreases in $\mathrm{B}$ and $\mathrm{T}$ cells in the lymphocyte population. It is likely that the increase of NK cells in method 1 was a reflection of the substantial reduction of $B$ and $\mathrm{T}$ cells due to extensive cell death. However, the possibility that the change in NK cells may have been a stochastic event cannot be excluded; compared with other cells, the percentage of NK cells exhibited the highest variability in different individuals. This is complicated further when age-dependent NK change is considered in children (17). With the exception of methods 1 and 2 , the observation that cryopreservation induced a percentage change in $\mathrm{CD}^{+} \mathrm{T}$ cells in lymphocytes or $\mathrm{CD}^{+} / \mathrm{CD}^{+}$single positive $\mathrm{T}$ cells in total $\mathrm{CD}^{+} \mathrm{T}$ cells may be the result of insufficient sampling as these changes are marginal.

Isolating lymphocytes using separation medium prior to freezing with standard cryoprotective medium (methods 5 and 6), conferred protection of cell viability regardless of whether HSA or FBS was used. The data revealed that the cells thawed from these two methods expressed intracellular IFN $\gamma$ and IL-2 (Fig. 4), but not those from methods 3 and 4, 
fresh (method 1-6)

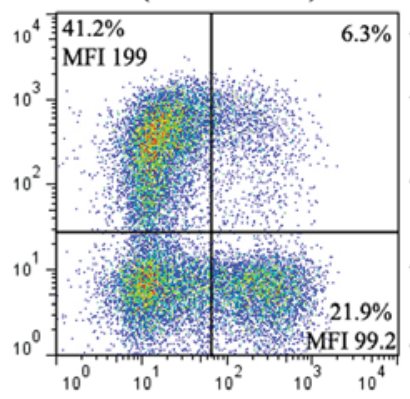

method 1

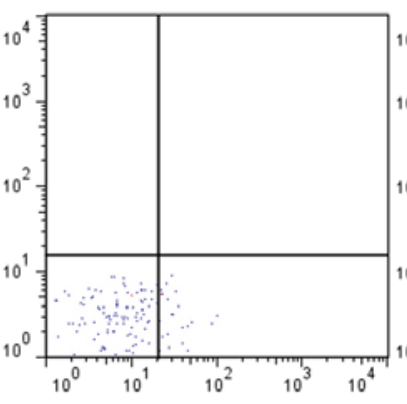

method 3

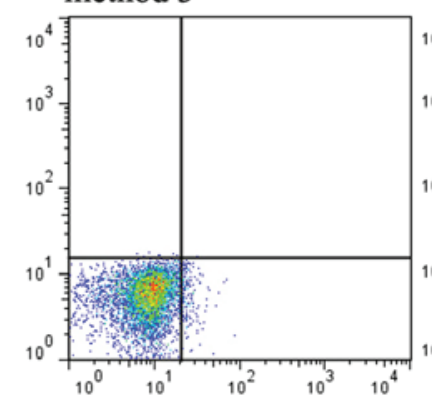

method 4

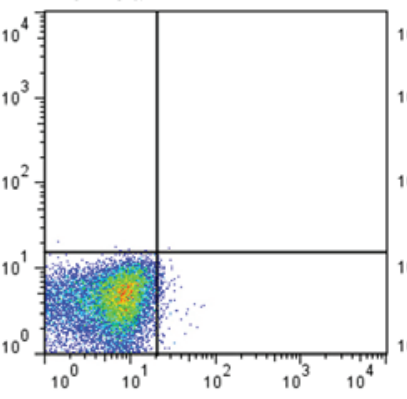

method 7

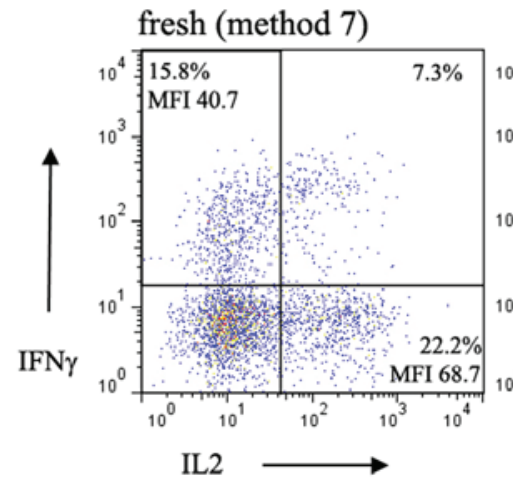

method 2

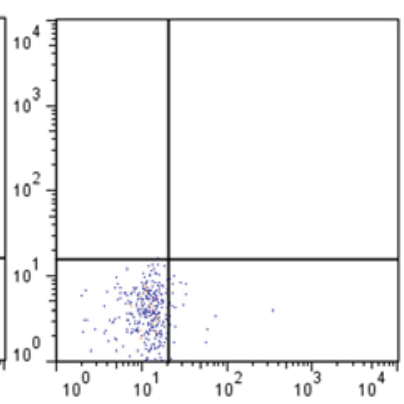

method 5
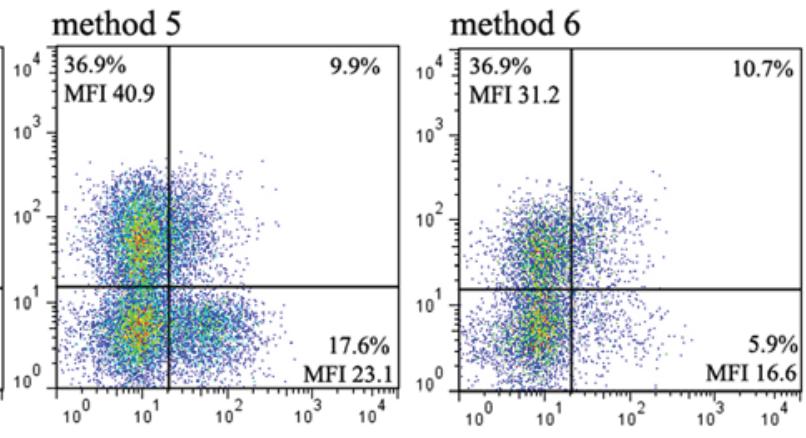

fresh (method 8)

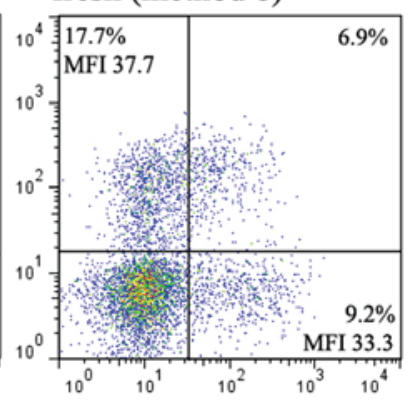

method 8

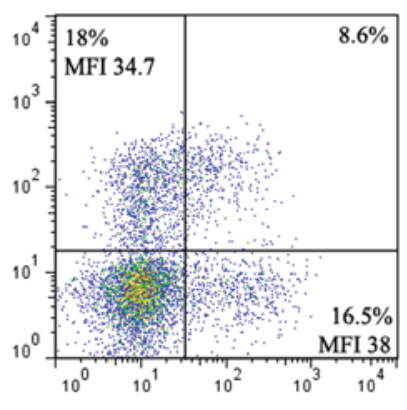

Figure 4. Lymphocyte cell function following cryopreservation. Lymphocytes in the blood sample were isolated with lymphocyte separation medium and cultured in U-bottom 96-well plates. Intracellular staining of IL-2 and IFN $\gamma$ were performed following stimulation of the cells with anti-CD3/CD28, PMA and ionomycin. Maintenance of cell function following cryopreservation was evaluated by comparing the intracellular expression levels of IL-2 and IFN $\gamma$ between fresh cultured lymphocytes and those thawed following different methods of cryopreservation. Methods 1 and 6 shared the same fresh sample. Results from methods 7 and 8 were compared with their respective fresh samples. In the representative flow diagrams, the percentages and MFIs of IFN $\gamma$ and IL-2 cells in lymphocytes gates were labeled. The percentage of IFN $\gamma^{+} \mathrm{IL}-2^{+}$double positive cells were also labeled. No IFN $\gamma$ or IL-2 $2^{+}$cells were detected following use of methods 1-4, therefore, the percentage and MFI were not labeled. At least four independent experiments were performed, with three samples of each method in independent experiments. IL-2, interleukin-2; IFN $\gamma$, interferon $\gamma$; MFI, mean fluorescence intensity.

which has been confirmed to maintain cell viability and surface marker expression. Commonly used hemolysin RBC lysis buffer (Optilyse C lysis solution) contains formaldehyde as a fixative. It is likely that the lymphocytes were partially fixed and that activation of the lymphocytes was inhibited by hemolysin treatment. This result provided experimental evidence that, when cell functional analysis is planned, the hemolysin lysis method is not suitable. When $\mathrm{NH}_{4} \mathrm{Cl}$ was used instead of hemolysin $\mathrm{RBC}$ lysis buffer in methods 7 and 8, the intracellular expression of IL-2 and IFN $\gamma$ recovered, compared with those in methods 3 and 4. Of note, as the majority of monocytes and granulocytes were lost in methods 5 and 6 , these two methods are not suggested for use if cells other than lymphocytes are the focus of interest (Fig. 1). In addition, 50\% of total lymphocytes were lost by cell isolation, which indicated this method is not suitable for experiments or clinical preparations requiring large quantities of cells.
The cryopreservative method with the optimal performance among the methods assessed in the present study was method 8 , in terms of the viability maintenance, surface marker expression and cell function of activated lymphocytes. Considering the HSA used in method 8 is cheaper than FBS and does not contain zoogenic substances, method 8 may be a cost-effective and safer cryopreservative approach for clinical applications in addition to experimental investigations.

The TCR repertoire, determined by specific antibodies recognizing different TCR subfamilies with variable V $\beta$ chains, is a useful index for antigen-specific responses, including infection, immunodeficiency or autoimmune diseases (18-20). However, in the present study, none of the cryopreservative methods were found to maintain the unbiased percentages of all the $24 \mathrm{~V} \beta$ subfamilies simul- 
A

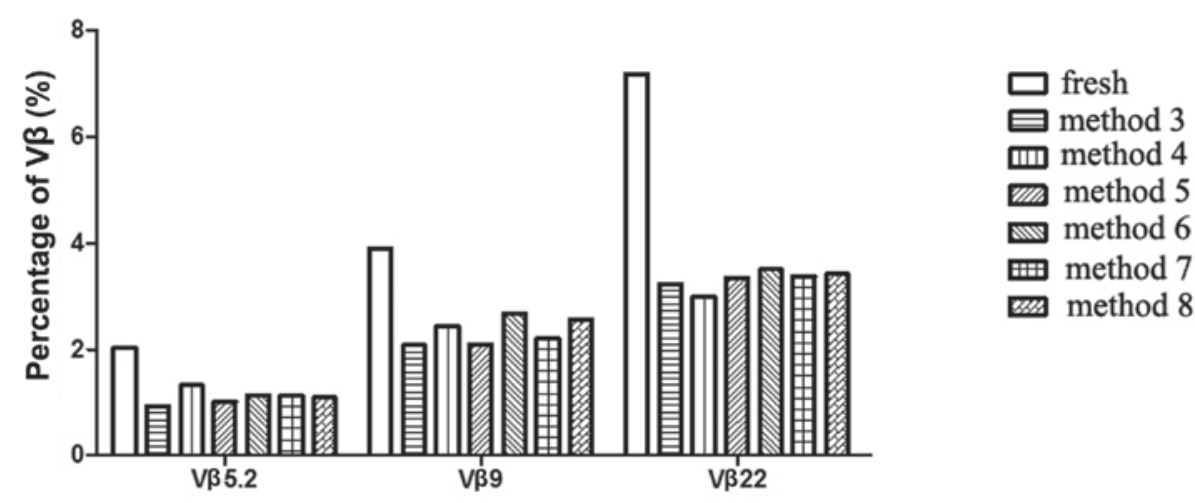

B

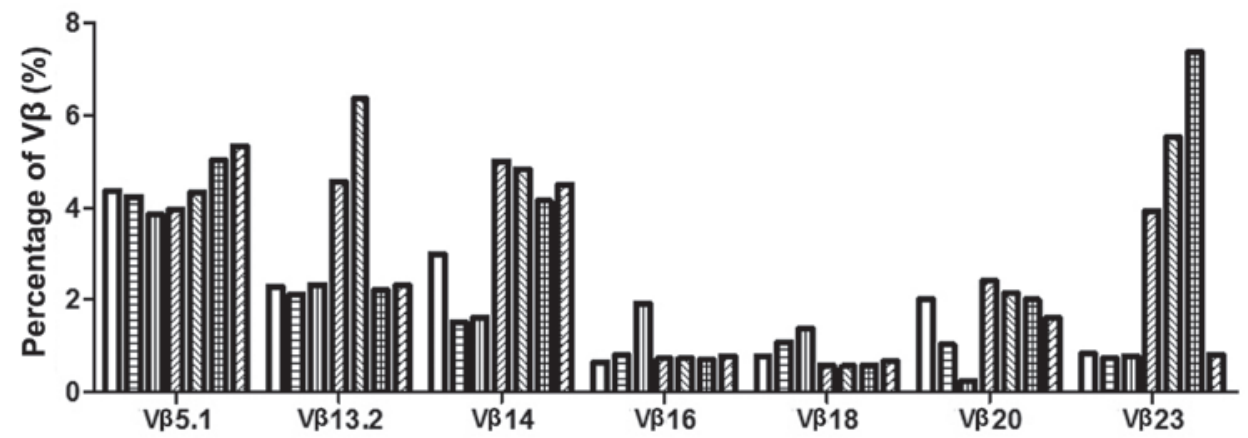

C
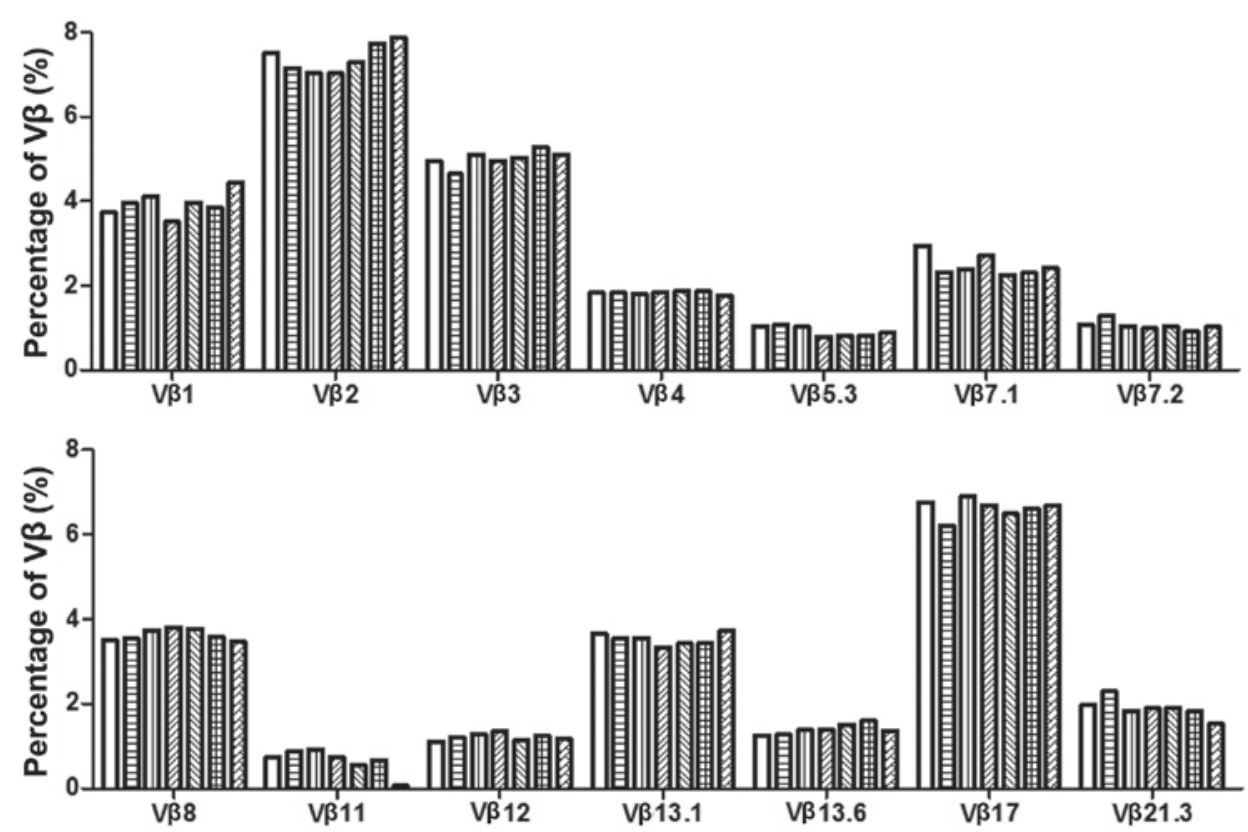

Figure 5. TCR V $\beta$ repertoire following cryopreservation. A total of 24 TCR V $\beta$ subgroups were detected using flow cytometry and their percentages in thawed samples following cryopreservation using the different methods were compared with their respective percentages prior to freezing (fresh). Changes in the percentages caused by freezing are summarized as three groups. (A) All freezing methods caused a decrease in the percentage of the TCR V $\beta$ subgroups indicated; (B) Variable method-dependent changes were observed in the percentages of the V $\beta$ subgroups indicated; (C) No freezing methods altered the percentages of the TCR V $\beta$ subgroups indicated. TCR V $\beta$, T cell receptor V $\beta$.

taneously. Therefore, method-dependent alterations in the percentages of particular $\mathrm{V} \beta$ subfamilies were found, which are misleading in forming conclusions on the TCR V $\beta$ repertoire. Accordingly, accurate evaluation of the TCR repertoire may be attained either by using fresh lymphocyte samples or using sequencing technology from DNA material $(21,22)$.

\section{Acknowledgements}

This study was supported by a startup research fund to Professor Jingang Gui from the Beijing Children's Hospital, a teaching hospital affiliated with Capital Medical University (Beijing, China). 


\section{References}

1. Chattopadhyay PK and Roederer M: Cytometry: Today's technology and tomorrow's horizons. Methods 57: 251-258, 2012.

2. Buhl T, Legler TJ, Rosenberger A, Schardt A, Schön MP and Haenssle HA: Controlled-rate freezer cryopreservation of highly concentrated peripheral blood mononuclear cells results in higher cell yields and superior autologous T-cell stimulation for dendritic cell-based immunotherapy. Cancer Immunol Immunother 61: 2021-2031, 2012.

3. Finn OJ: Cancer Immunology. N Engl J Med 358: 2704-2715, 2008.

4. Curran KJ, Pegram HJ and Brentjens RJ: Chimeric antigen receptors for $\mathrm{T}$ cell immunotherapy: Current understanding and future directions. J Gene Med 14: 405-415, 2012.

5. Antoniewicz-Papis J, Lachert E, Woźniak J, Janik K and Łętowska M: Methods of freezing cord blood hematopoietic stem cells. Transfusion 54: 194-202, 2014.

6. Stevens VL, Patel AV, Feigelson HS, Rodriguez C, Thun MJ and Calle EE: Cryopreservation of whole blood samples collected in the field for a large epidemiologic study. Cancer Epidemiol Biomarkers Prev 16: 2160-2163, 2007.

7. Germann A, Schulz JC, Kemp-Kamke B, Zimmermann H and von Briesen H: Standardized serum-free cryomedia maintain peripheral blood mononuclear cell viability, recovery, and antigen-specific T-cell response compared to fetal calf serum-based medium. Biopreserv Biobank 9: 229-236, 2011.

8. Dijkstra-Tiekstra MJ, Setroikromo AC, Kraan M, Gkoumassi E and de Wildt-Eggen J: Optimization of the freezing process for hematopoietic progenitor cells: Effect of precooling, initial dimethyl sulfoxide concentration, freezing program, and storage in vapor-phase or liquid nitrogen on in vitro white blood cell quality. Transfusion 54: 3155-3163, 2014.

9. Petrenko YA, Rogulska OY, Mutsenko VV and Petrenko AY: A sugar pretreatment as a new approach to the Me2SO- and xeno-free cryopreservation of human mesenchymal stromal cells. Cryo Letters 35: 239-246, 2014.

10. Hreinsson J, Zhang P, Swahn ML, Hultenby K and Hovatta O: Cryopreservation of follicles in human ovarian cortical tissue. Comparison of serum and human serum albumin in the cryoprotectant solutions. Hum Reprod 18: 2420-2428, 2003.

11. Wiegering V, Eyrich M, Wunder C, Günther H, Schlegel PG and Winkler B: Age-relate changes in intracellular cytokine expression in healthy children. Eur Cytokine Netw 20: 75-80, 2009.
12. Bunders $M$, Cortina-Borja $M$ and Newell ML; European Collaborative Study: Age-related standards for total lymphocyte, CD4+ and CD8+ T cell counts in children born in Europe. Pediatr Infect Dis J 24: 595-600, 2005.

13. Wu J, Liu D, Tu W, Song W and Zhao X: T-cell receptor diversity is selectively skewed in T-cell populations of patients with Wiskott-Aldrich syndrome. J Allergy Clin Immunol 135: 209-216, 2015

14. Tzifi F, Kanariou M, Tzanoudaki M, Mihas C, Paschali E, Chrousos $\mathrm{G}$ and Kanaka-Gantenbein C: Flow cytometric analysis of the CD4+ TCR V $\beta$ repertoire in the peripheral blood of children with type 1 diabetes mellitus, systemic lupus erythematosus and age-matched healthy controls. BMC Immunol 14: 33, 2013.

15. Attaf M, Huseby E and Sewell AK: $\alpha \beta$ T cell receptors as predictors of health and disease. Cell Mol Immunol 12: 391-399, 2015.

16. Gil A, Yassai M, Naumov Y and Selin L: Narrowing of human influenza $A$ virus-specific T cell receptor $\alpha$ and $\beta$ repertoires with increasing age. J Virol 89: 4102-4116, 2015.

17. Osugi Y,Hara J, Kurahashi H, Sakata N, Inoue M, Yumura-Yagi K, Kawa-Ha K, Okada S and Tawa A: Age-related changes in surface antigens on peripheral lymphocytes of healthy children. Clin Exp Immunol 100: 543-548, 1995.

18. Chen H, Ndhlovu ZM, Liu D, Porter LC, Fang JW, Darko S, Brockman MA, Miura T, Brumme ZL, Schneidewind A, et al: TCR clonotypes modulate the protective effect of HLA class I molecules in HIV-linfection. Nat Immunol 13: 691-700, 2012.

19. Aleman K, Noordzij JG, de Groot R, van Drogen JJ and Hartwig NG: Reviewing Omenn syndrome. Eur J Pediatr 160: 718-725, 2001.

20. Somma P, Ristori G, Battistini L, Cannoni S, Borsellino G, Diamantini A, Salvetti M, Sorrentino R and Fiorillo MT: Characterization of CD81 T cell repertoire in identical twins discordant and concordant for multiple sclerosis. J Leukoc Biol 81: 696-710, 2007.

21. Fang H, Yamaguchi R, Liu X, Daigo Y, Yew PY, Tanikawa C, Matsuda K, Imoto S, Miyano S and Nakamura Y: Quantitative $\mathrm{T}$ cell repertoire analysis by deep cDNA sequencing of $\mathrm{T}$ cell receptor $\alpha$ and $\beta$ chains using next-generation sequencing (NGS). Oncoimmunology 3: e968467, 2015.

22. Gao F and Wang K: Ligation-anchored PCR unveils immune repertoire of TCR-beta from whole blood. BMC Biotechnol 15: 39, 2015. 